\title{
Spin-Dephasing Anisotropy for Electrons in a Diffusive Quasi-1D GaAs Wire
}

\author{
J. Liu • T. Last · E.J. Koop · S. Denega • B.J. van Wees • \\ C.H. van der Wal
}

Received: 13 February 2009 / Accepted: 11 August 2009 / Published online: 19 September 2009

(C) The Author(s) 2009. This article is published with open access at Springerlink.com

\begin{abstract}
We present a numerical study of dephasing of electron spin ensembles in a diffusive quasi-one-dimensional GaAs wire due to the D'yakonov-Perel' spin-dephasing mechanism. For widths of the wire below the spin precession length and for equal strength of Rashba and linear Dresselhaus spin-orbit fields a strong suppression of spin-dephasing is found. This suppression of spin-dephasing shows a strong dependence on the wire orientation with respect to the crystal lattice. The relevance for realistic cases is evaluated by studying how this effect degrades for deviating strength of Rashba and linear Dresselhaus fields, and with the inclusion of the cubic Dresselhaus term.
\end{abstract}

Keywords Semiconductor spintronics - Two-dimensional electron gas $\cdot$ Spin-orbit coupling $\cdot$ Spin relaxation

\section{Introduction}

The ability to maximize the spin-dephasing time $T_{2}^{*}$ of an electron spin ensemble is one of the key issues for developing semiconductor-based spintronic devices [1,2]. However, in all III-V semiconductor materials spin ensembles rapidly dephase due to the D'yakonov-Perel' (DP) spin-dephasing mechanism [3-5]. For the case of electron ensembles in a heterojunction two-dimensional electron gas (2DEG), two distinct contributions to DP spin-dephasing have to be considered: the inversion asymmetry of the confining potential (structural inversion asymmetry) and the bulk inversion

J. Liu · T. Last (凶) · E.J. Koop · S. Denega · B.J. van Wees ·

C.H. van der Wal

Physics of Nanodevices Group, Zernike Institute for Advanced

Materials, University of Groningen, Nijenborgh 4,

9747AG Groningen, The Netherlands

e-mail: t.last@rug.nl asymmetry of the crystal lattice. The former results in an effective Rashba field and the latter in an effective Dresselhaus field, which includes linear and cubic contributions [3-8]:

$\vec{B}_{R}=C_{R}\left(\hat{x} k_{y}-\hat{y} k_{x}\right)$,

$\vec{B}_{D 1}=C_{D 1}\left(-\hat{x} k_{x}+\hat{y} k_{y}\right)$,

$\vec{B}_{D 3}=C_{D 3}\left(\hat{x} k_{x} k_{y}^{2}-\hat{y} k_{x}^{2} k_{y}\right)$,

where $\hat{x}, \hat{y}$ are the unit vectors along the [100] and [010] crystal directions; $k_{x}, k_{y}$ are the components of the in-plane wave vector; and $C_{R, D 1, D 3}$ are the spin-orbit coupling parameters. The total effective spin-orbit field $\vec{B}_{\text {eff }}$ is the vector sum of all three contributions. For 2D and quasi-1D electron systems, the direction and magnitude of these effective spin-orbit fields can be illustrated as arrows on the Fermi circle. Figure 1 presents this for selected points in the 2D momentum space, for the Rashba (a) and linear Dresselhaus (b) field alone, and their sum (c) for the case of equal strength of Rashba and linear Dresselhaus field. In contrast to the individual cases (Fig. 1 (a), (b)), the magnitude of the vector sum shows a strong anisotropy in momentum space (Fig. 1 (c)). This already suggests that spin-dephasing in very narrow wires in which electron motion is restricted to the [110] direction, can be strongly reduced as compared to free 2DEG or such wires oriented along other crystal directions. However, it is harder to analyze whether such a dephasing anisotropy also occurs for wider quasi-1D wires, where the motion in the 2DEG plane is still completely random and diffusive, but where the width of the wire is less than length scales as the spin precession length or the mean free path. For the latter case the transport regime could be named quasi-ballistic, but we consider the case of a large ensemble where transport along the wire is still diffusive, and where the wire width in the 2DEG plane is much larger 


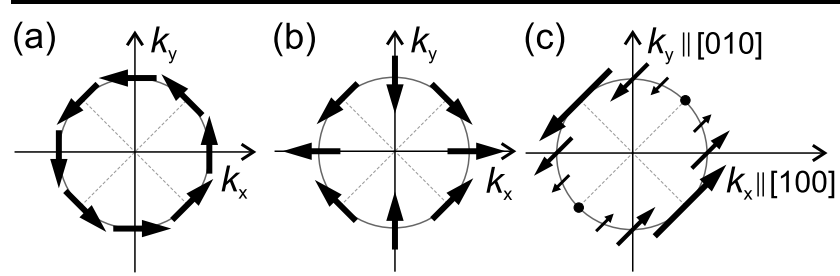

Fig. 1 A schematic representation of the direction and magnitude of the effective magnetic field for selected points in a two-dimensional $k$-space, sketched for (a) the Rashba field, (b) the linear Dresselhaus field, and (c) the symmetric case of the sum of equal Rashba and linear Dresselhaus field. Both the magnitude and direction are depicted as arrows on the Fermi circle with radius $k_{F}$ in the $\left(k_{x}, k_{y}\right)$-plane

than the regime of quantum confinement. Initial studies of such spin-dephasing anisotropy include a recent experiment [9] on wires, and theoretical work on drifting ensembles in free 2DEG [10]. However, until now, the most emphasis was on work related to the spin field-effect transistor [11], using InAs-based systems or highly asymmetrical heterojunctions, where structural inversion asymmetry dominates the spinorbit interaction [12-16].

We report here how the D'yakonov-Perel' spin-dephasing mechanism can be strongly suppressed in diffusive quasi-1D electron systems based on GaAs heterojunction material, for which Rashba and linear Dresselhaus spin-orbit contributions can be indeed of comparable magnitude $[5,9]$. The dephasing is studied for spin ensembles initially aligned perpendicular to the plane of the wire ([001] direction). This situation reflects the method of preparing and interrogating a spin population via optical pump-probe techniques [2]. Our numerical calculation is first performed for conditions with equal Rashba and linear Dresselhaus contributions and the cubic Dresselhaus term set to zero. For widths of the diffusive quasi-1D wires smaller than the spin precession length, the DP spin-dephasing mechanism can be strongly suppressed and the spin-dephasing time $T_{2}^{*}$ is considerably enhanced if the wire is aligned along the direction of zero effective spin-orbit field. Moreover, we want to point out that the value of our numerical tool lies in the opportunity to study such phenomena also for more realistic conditions. Thus, we can study how breaking the equality of the Rashba and linear Dresselhaus spin-orbit fields, or adding the cubic Dresselhaus term, leads to a degradation of the spindephasing anisotropy.

\section{Method}

We apply a Monte Carlo method [17] to study the temporal evolution of the normalized spin orientation (average spin expectation value) in an elongated quasi-1D wire. Our numerical tool is based on a semiclassical approach. We use a classical description for the electron motion, and a quantum mechanical description of the dynamics of the electron spin. The wire is treated as a rectangular box of aspects $1 \mu \mathrm{m}$ and $200 \mu \mathrm{m}$. The electron density and mobility are set to $4 \cdot 10^{15} \mathrm{~m}^{-2}$ and $100 \mathrm{~m}^{2} / \mathrm{Vs}$, which are typical values for a GaAs/AlGaAs heterojunction material. All electrons are assumed to have the same Fermi velocity $v_{F}$ of $2.7 \cdot 10^{5} \mathrm{~m} / \mathrm{s}$. This is a valid approximation for $k_{B} T$, $\Delta E_{Z, S O} \ll E_{F}$ (with respect to the bottom of the conduction band), where $\Delta E_{Z, S O}$ is the Zeeman splitting due to the spin-orbit fields alone. Electron-electron interaction and inelastic scattering mechanisms are neglected.

The electron is regarded as a point particle which moves on a classical trajectory between scattering events on impurities (randomly determined at a rate to obtain an average scatter time of $38 \mathrm{ps}$ ) yielding diffusive behavior in the ensemble (electron mean free path $L_{p}=v_{F} \tau_{p}=10 \mu \mathrm{m}$ ) and specular scattering on the edges of the wire. For each electron moving on such a ballistic trajectory we calculate the spin evolution in the effective spin-orbit fields quantum mechanically, and we then take the ensemble average on a set of electrons with random initial position and momentum direction.

Within a straight ballistic segment of an electron trajectory the spin rotates around $\vec{B}_{\text {eff }}$ over a precession angle given by

$\phi_{\text {prec }}=\frac{g \mu_{B}\left|\vec{B}_{\text {eff }}\right|}{\hbar} t$,

where $\hbar$ is the reduced Planck's constant and $t$ the time of traveling through the segment. The spin rotation operator $\widehat{U}$ for rotation over the precession angle $\phi_{\text {prec }}$ about the direction $\vec{u}$ (unit vector) of the effective magnetic field $\vec{B}_{\text {eff }}$ is obtained by (see e.g. [18])

$$
\begin{aligned}
\widehat{U} & =\exp \left(-i \frac{\phi_{\text {prec }}}{2} \vec{\sigma} \vec{u}\right) \\
& =\left(\begin{array}{cc}
\cos \frac{\phi_{\text {prec }}}{2}-i u_{z} \sin \frac{\phi_{\text {prec }}}{2} & -\left(i u_{x}+u_{y}\right) \sin \frac{\phi_{\text {prec }}}{2} \\
-\left(i u_{x}-u_{y}\right) \sin \frac{\phi_{\text {prec }}}{2} & \cos \frac{\phi_{\text {prec }}}{2}+i u_{z} \sin \frac{\phi_{\text {prec }}}{2}
\end{array}\right),
\end{aligned}
$$

where $\vec{\sigma}$ represents the vector of Pauli spin matrices $(x, y$, $z$ components of the spin). $\widehat{U}$ acting on a spin state $\left|\Psi_{\text {initial }}\right\rangle$ at the beginning of a ballistic trajectory yields the spin state $\left|\Psi_{\text {final }}\right\rangle=\widehat{U}\left|\Psi_{\text {initial }}\right\rangle$ at the end of the trajectory. Thus, we can follow the spin state of each electron (labeled $i$ ), and we use this to define a semiclassical spin vector to present its orientation, $\vec{S}_{i}=\left(\left\langle S_{x}\right\rangle_{i},\left\langle S_{y}\right\rangle_{i},\left\langle S_{z}\right\rangle_{i}\right)$ from its spin expectation values in $x, y, z$ directions.

For an electron experiencing multiple scattering events, the orientation of the effective magnetic field is changed at 
each scattering event. For each trajectory a rotation is applied. Once a scattering event takes place, the wave vector state is updated, $\vec{B}_{\text {eff }}$ is recalculated based on the new wave vector, resulting in a new rotation operator, and the evolution of the spin state will carry on. For spin ensembles, the randomization will bring a reduction of the normalized spin orientation (average spin expectation value) $\left\langle S_{r}\right\rangle=\left|\left(\sum_{i=1}^{N} \vec{S}_{i}\right) / N\right|$ for the ensemble. $\left\langle S_{r}\right\rangle$ is obtained by averaging over an ensemble of $N=1000$ spins, independently of their positions within the system, and calculated as a function of time. We choose to study the spin coherence in the ensemble here as $\left\langle S_{r}\right\rangle$. The advantage is that $\left\langle S_{r}\right\rangle$ gives the magnitude of the residual spin orientation in the direction that is maximal (automatically evaluating the envelope in case the ensemble average shows precession). However, in the present study, without externally applied fields, there was no development of average spin orientation in the $x$ and $y$ directions, and the decay of $\left\langle S_{r}\right\rangle$ always equaled the decay of ensemble average $\left\langle S_{z}\right\rangle=\left(\sum_{i=1}^{N}\left\langle S_{z}\right\rangle_{i}\right) / N$. The spindephasing time $T_{2}^{*}$ of the ensemble is defined as the decay time over which $\left\langle S_{r}\right\rangle$ reduces to $1 /$ e of its initial value. Note, however, that decay traces of $\left\langle S_{r}\right\rangle$ were not always monoexponential in our simulations.

\section{Results and Discussion}

The temporal evolution of the normalized spin orientation $\left\langle S_{r}\right\rangle$ is studied for out-plane initial spin states oriented along the [001] direction. The effective spin-orbit field resulting from equal magnitudes of the Rashba and linear Dresselhaus fields is always parallel to the [110] axis $\left(C_{R}=C_{D 1}=\right.$ $\left.-1.57 \cdot 10^{-8} \mathrm{Tm},[5]\right)$. These values of the spin-orbit parameters give rise to an average spin precession length of about $3 \mu \mathrm{m}$. The width of our wire of $1 \mu \mathrm{m}$ is chosen to be smaller than this length scale.

The inset of Fig. 2 shows the temporal evolution for five different orientations of the quasi-1D wire with respect to the [100] direction. A distinct anisotropy of the spin-dephasing times is observed. The peak value of $T_{2}^{*}$ is reached when the wire is oriented exactly along the [110] direction. It is found to be in excess of $10^{4} \mathrm{ps}$. This agrees with the result $T_{2}^{*}=\infty$, which can be derived analytically for this ideal case $[19,20]$. Yet, a deviation of only $5^{\circ}$ in the wire orientation with respect to the [110] direction leads to a reduction of $T_{2}^{*}$ of more than an order of magnitude towards a value of about 200 ps. For angles close to [110] $\pm 15^{\circ}$ the spin-dephasing time drops already to $43 \mathrm{ps}$ and it reaches a minimum of $9 \mathrm{ps}$ for wires oriented along the [110] and [1 $1 \overline{1} 0]$ direction. A detailed summary of these findings is presented in Fig. 2 where the extracted spin-dephasing times are plotted as a function of the wire orientation. This plot reveals the strong anisotropy of $T_{2}^{*}$ with respect to the crystal axes.

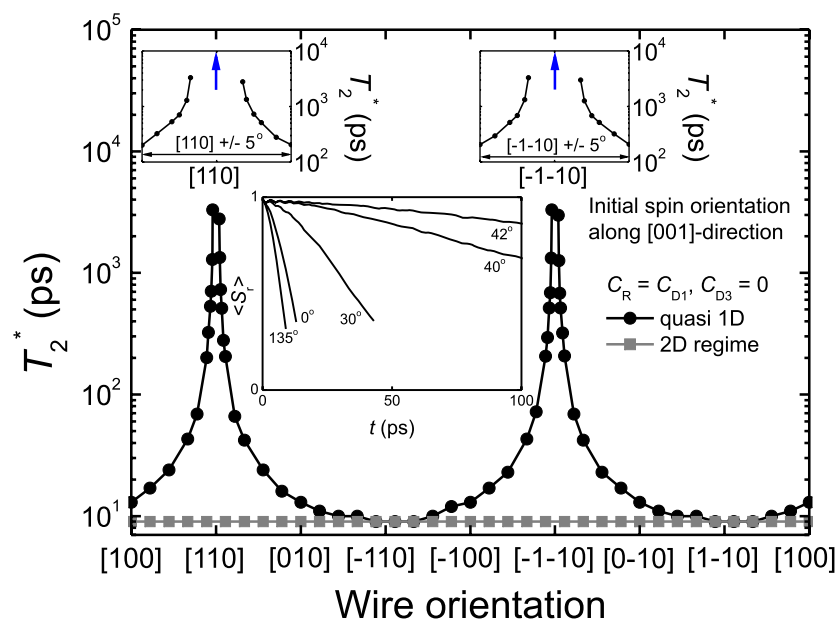

Fig. 2 The spin-dephasing time $T_{2}^{*}$ of a spin ensemble is plotted as a function of the wire orientation with respect to the [100] lattice direction. The ensemble is initially oriented along the [001] direction. $T_{2}^{*}$ is strongly enhanced for a quasi-1D wire oriented in the [110] direction (black) as compared to an ensemble in a $2 \mathrm{D}$ system (gray). The arrows (in the top insets, horizontal axes span $\pm 5^{\circ}$ ) for data at $45^{\circ}$ and $225^{\circ}$ indicate that here $T_{2}^{*}$ is larger than could be calculated $\left(C_{R}=C_{D 1}=-1.57 \cdot 10^{-8} \mathrm{Tm}, C_{D 3}=0\right)$. Inset: Ensemble spin expectation value $\left\langle S_{r}\right\rangle$ as a function of time for different wire orientations

The anisotropy of $T_{2}^{*}$ is directly related to the motion of single electrons within the ensemble. Specular edge scattering implies that electrons with a solely transverse momentum component to the wire orientation (traveling less than the spin precession length between scattering events) almost do not contribute to the spin-dephasing because of motional narrowing. Only electrons with a strong momentum component longitudinal to the wire orientation are contributing to the dephasing of the spin ensemble. This results in the strong enhancement of $T_{2}^{*}$ for wires in the [110] direction. Spin-dephasing times for non-confined spin ensembles (wire width taken much larger than the mean free path and spin precession length) are also calculated (gray, Fig. 2). In contrast to the quasi-1D wire case, no spin-dephasing anisotropy is found. We investigated the crossover from 2D to quasi-1D behavior for a wide range of values for the spin precession length and mean free path (with respect to the wire width), and found that the spin precession length is the crucial length scale which is governing this crossover.

Next, we discuss whether this distinct spin-dephasing anisotropy in quasi-1D wires (with a strong enhancement of $T_{2}^{*}$ for wires in the [110] direction as a fingerprint) can be maintained under more realistic circumstances. First we study the effect of adding the cubic Dresselhaus term on the enhancement of $T_{2}^{*}$. Secondly, the influence of deviating strength of Rashba and linear Dresselhaus fields will be discussed. To avoid the difficulty that $T_{2}^{*}$ cannot be calculated for $45^{\circ}$ with respect to the [100] direction within reasonable calculation time, we take $43^{\circ}$ as a test case, as this already 

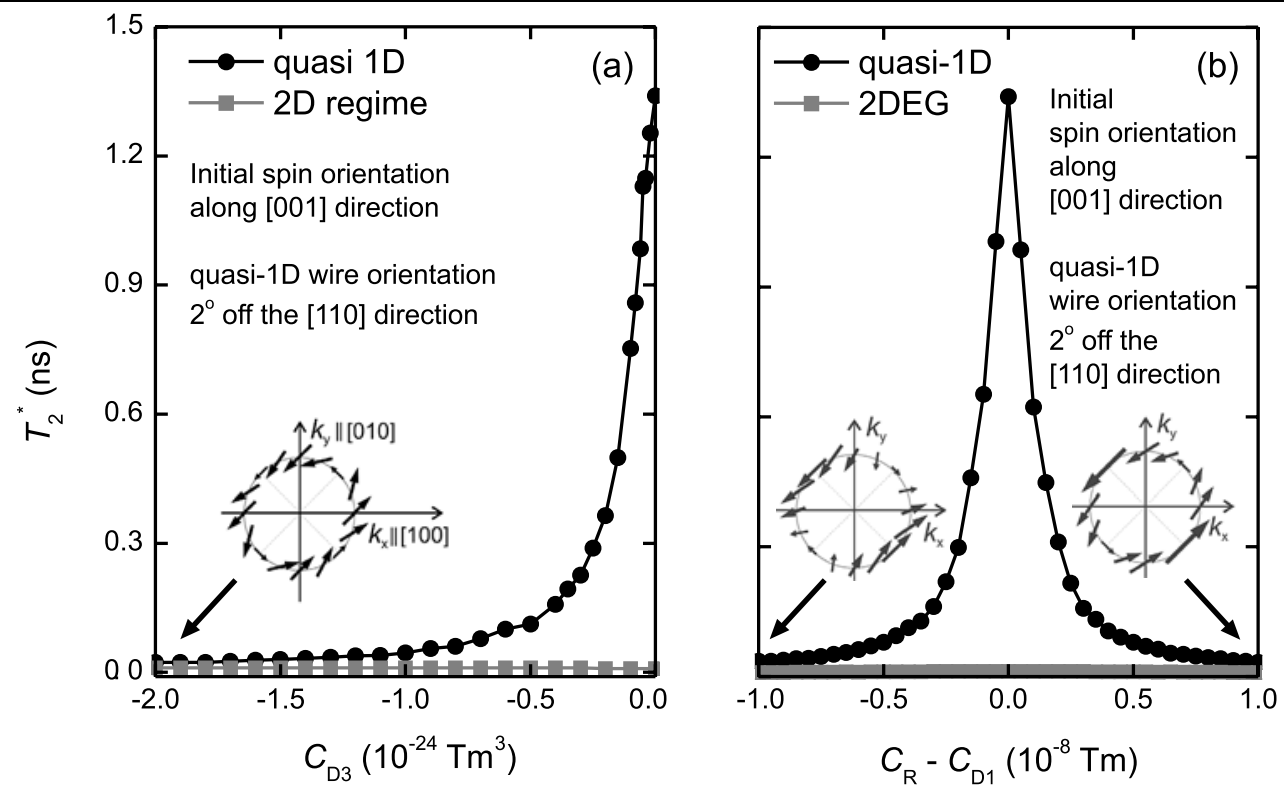

Fig. 3 Data shows how the $T_{2}^{*}$ enhancement of Fig. 2 reduces when the cubic Dresselhaus term is added and when the symmetry between $C_{R}$ and $C_{D 1}$ is lifted. The initial spin state is chosen to be along [001] direction and the quasi-1D wire is set at $43^{\circ}$ with respect to the [100] direction. The case of $43^{\circ}$ wire orientation (rather than $45^{\circ}$ ) avoids the need to deal in calculations with extremely long $T_{2}^{*}$ for the symmet-

shows a very strong $T_{2}^{*}$ enhancement, while $T_{2}^{*}$ is limited to nanoseconds. Hence, the calculated spin-dephasing times in the following part can be seen as lower bounds. $T_{2}^{*}$ for the exact [110] direction is expected to be distinctively higher.

The effect of the additional cubic Dresselhaus term on the spin-dephasing time in the quasi-1D wire is presented in Fig. 3 (a). Again, the 2D case is plotted as a reference (gray). Without the cubic Dresselhaus term the calculation results in a $T_{2}^{*}$ of $1.3 \mathrm{~ns}$. However, the spin-dephasing time is decreasing rapidly with increasing Dresselhaus parameter. For $C_{D 3}=-7 \cdot 10^{-25} \mathrm{Tm}^{3}, T_{2}^{*}$ is already reduced to $78 \mathrm{ps}$. To estimate whether the spin-dephasing anisotropy is still present in a more realistic situation, experimentally deduced parameters are applied for comparison with our calculations. In [5], a value of $C_{D 3}=-1.18 \cdot 10^{-24} \mathrm{Tm}^{3}$ is evaluated, which results in a $T_{2}^{*}$ of only $40 \mathrm{ps}-\mathrm{a}$ value which is less than an order of magnitude higher than the value calculated for the 2D case. This points to the conclusion that the cubic Dresselhaus term nearly annihilates the spin-dephasing anisotropy. However, $C_{D 3}$ depends strongly on the electron density of the system. For samples with lower densities, $C_{D 3}$ is orders of magnitude smaller [5, 21, 22]. Considering those values, it turns out that there is a much weaker decay of the peak value of the spin-dephasing time, even when the cubic Dresselhaus term is included.

Finally, $T_{2}^{*}$ is investigated for deviating Rashba and linear Dresselhaus contributions. This dependence is summa- ric case of $C_{R}=C_{D 1}$ and $C_{D 3} \approx 0$, while still clearly showing the 1D $T_{2}^{*}$ enhancement. (a) The effect of the cubic Dresselhaus term on $T_{2}^{*}$ is plotted for the 2D case (gray) and for the quasi-1D case (black) $\left(C_{R}=C_{D 1}=-1.57 \cdot 10^{-8} \mathrm{Tm}\right)$. (b) $T_{2}^{*}$ is plotted here against the difference $C_{R}-C_{D 1}$, at the fixed value of $C_{D 1}=-1.57 \cdot 10^{-8} \mathrm{Tm}$ for the $2 \mathrm{D}$ case (gray) and for the quasi-1D case (black) $\left(C_{D 3}=0\right)$

rized in Fig. 3 (b), where $T_{2}^{*}$ is plotted as a function of the difference in strength of the Rashba and linear Dresselhaus parameter, $\left(C_{R}-C_{D 1}\right)$, in the interval $\pm 1 \cdot 10^{-8} \mathrm{Tm}$ for $C_{D 1}$ fixed at $-1.57 \cdot 10^{-8} \mathrm{Tm}$. At $C_{R}-C_{D 1}=0$ this results in the previously calculated $T_{2}^{*}$ of around $1.3 \mathrm{~ns}$. With either increasing $\left|C_{R}\right|$ or increasing $\left|C_{D 1}\right|, T_{2}^{*}$ is decaying equally fast. $C_{R}$ and $C_{D 1}$ taken from [5] result in a difference $C_{R}-C_{D 1}$ of $0.4 \cdot 10^{-8} \mathrm{Tm}$. For this value our calculated $T_{2}^{*}$ is already considerably reduced to about $110 \mathrm{ps}$. However, this spin-dephasing time is still an order of magnitude higher than the one resulting from the $2 \mathrm{D}$ case, and in addition, $C_{R}$ can be tuned with a gate or heterostructure design to equalize it to the linear Dresselhaus field. Therefore, in summary, it can be stated that the spin-dephasing anisotropy, which is very distinct for $C_{R}$ and $C_{D 1}$ exactly equal, can still prevail under less ideal conditions.

\section{Conclusion}

A useful numerical tool is developed for studying spindephasing in device structures due to the D'yakonov-Perel' spin-dephasing mechanism. The Rashba, linear and cubic Dresselhaus contributions can be taken into account. With this tool it was demonstrated that quasi-1D wires (narrower than the spin precession length, but with diffusive 2D motion for the electron ensemble) can show very clear signatures 
of spin-dephasing anisotropy, with a strong suppression of spin-dephasing for wires in the [110] crystal direction.

Acknowledgements We acknowledge financial support from the Dutch Foundation for Fundamental Research on Matter (FOM), the Netherlands Organization for Scientific Research (NWO) and by the Nanotechnology Network in the Netherlands (NanoNed).

Open Access This article is distributed under the terms of the Creative Commons Attribution Noncommercial License which permits any noncommercial use, distribution, and reproduction in any medium, provided the original author(s) and source are credited.

\section{References}

1. Awschalom, D.D., Loss, D., Samarth, N. (eds.): Semiconductor Spintronics and Quantum Computation. Springer, Berlin (2002). For a review

2. Kikkawa, J.M., Awschalom, D.D.: Phys. Rev. Lett. 80, 4313 (1998)

3. D'yakonov, M.I., Kachorovskii', V.Yu.: Fiz. Tekh. Poluprovodn. (S.-Peterburg) 20, 178 (1986)

4. D’yakonov, M.I., Kachorovskii', V.Yu.: Sov. Phys. Semicond. 20, $110(1986)$

5. Miller, J.B., Zumbühl, D.M., Marcus, C.M., Lyanda-Geller, Y.B., Goldhaber-Gordon, D., Campman, K., Gossard, A.C.: Phys. Rev. Lett. 90, 076801 (2003)
6. Bychkov, Y.A., Rashba, E.I.: Pis'ma Zh. Eksp. Teor. Fiz. 39, 66 (1984)

7. Bychkov, Y.A., Rashba, E.I.: JETP Lett. 39, 78 (1984)

8. Lommer, G., Malcher, F., Rössler, U.: Phys. Rev. B 32, 6965 (1985)

9. Frolov, S.M., Venkatesan, A., Yu, W., Lüscher, S., Wegscheider, W., Folk, J.A.: arXiv:0801.4021

10. Duckheim, M., Loss, D.: Phys. Rev. B 75, 201305(R) (2007)

11. Datta, S., Das, B.: Appl. Phys. Lett. 56, 665 (1990)

12. Kiselev, A.A., Kim, K.W.: Phys. Rev. B 61, 13115 (2000)

13. Pareek, T.P., Bruno, P.: Phys. Rev. B 65, 241305(R) (2002)

14. Mireles, F., Kirczenow, G.: Phys. Rev. B 64, 024426 (2001)

15. Pramanik, S., Bandyopadhyay, S., Cahay, M.: Phys. Rev. B 68, 075313 (2003)

16. Holleitner, A.W., Sih, V., Myers, R.C., Awschalom, D.D.: Phys. Rev. Lett. 97, 036805 (2006)

17. Koop, E.J., van Wees, B.J., van der Wal, C.H.: arXiv:0804.2968

18. Cohen-Tannoudji, C., Diu, B., Laloë, F.: Quantum Mechanics, vol. 1, p. 691. Wiley, New York (1977)

19. Folk, J.A.: private communication

20. Liu, J., et al. (in preparation)

21. Jusserand, B., Richards, D., Allan, G., Etienne, B.: Phys. Rev. B 51, 4707 (1995)

22. Richards, D., Jusserand, B., Peric, H., Etienne, B.: Phys. Rev. B 47, 16028 (1993) 